\title{
How to teach Quantum Mechanics
}

\author{
Oliver Passon \\ Fachbereich Physik, University of Wuppertal \\ Postfach 100 127, 42097 Wuppertal, Germany \\ E-mail: Oliver.Passon@cern.ch
}

In the spirit and style of John S. Bell's well known paper on How to Teach Special Relativity [1] it is argued, that a "Bohmian pedagogy" provides a very useful tool to illustrate the relation between classical and quantum physics and illuminates the peculiar features of the latter.

The paper by Bell on How to Teach Special Relativity introduces the subject with the following remark:

I have for long thought that if I had the opportunity to teach this subject, I would emphasize the continuity with earlier ideas. Usually it is the discontinuity which is stressed, the radical break with more primitive notions of space and time. Often the result is to destroy completely the confidence of the student in perfectly sound and useful concepts already acquired. ${ }^{1}$

In the following Bell gives an account of the Lorentzian interpretation of relativistic effects, in which the Lorentz transformations are explained by a dynamical mechanism (including the assumption of an - though undetectable - aether) rather than derived from postulates as in the approach of Ein$\operatorname{stein}^{2}$. Bell concludes that teaching relativity can benefit from what he calls a "Lorentzian pedagogy", i.e. a presentation of the Lorentzian viewpoint, since "the longer road sometimes gives more familiarity with the country".

Our note tries to translate this conclusion into the context of quantum theory, where Bohmian mechanics ${ }^{3}$ can serve a similar purpose as the

\footnotetext{
${ }^{1}$ Notes are to be ignored in a first reading

${ }^{2}$ How closely Bell actually stuck to Lorentz's thinking is debatable [7] but does not matter too much in our context.

${ }^{3}$ Bohmian mechanics is frequently referred to as de Broglie-Bohm theory, since Louis de Broglie had similar ideas already in 1927. David Bohm's work in 1952 [8] was done independently.
} 
Lorentzian aether theory with respect to special relativity. The similarities between both theories are in fact striking: (i) like the Lorentz interpretation, Bohmian mechanics derives the key ingredient (namely Born's probability rule) rather than postulating it, (ii) both, the Lorentz interpretation and Bohmian mechanics, make the same experimental predictions as their respective standard theories, while postulating undetectable entities, (iii) both were appreciated by John S. Bell, and finally, (iv) like the Lorentz interpretation, Bohmian mechanics emphasizes the continuity with earlier ideas more than the radical break with more primitive notions of (in this case) particles and matter.

Within Bohmian mechanics particles keep on moving on trajectories, while the quantum mechanical interference phenomena come about due to the role of the wavefunction as a "guiding field". At the same time this theory is extremely simple! As a starting point one may take the classical relation between an arbitrary current $(j)$, the related density $(\rho)$ and the velocity field $(v=d x / d t)^{4}$ :

$$
j=v \cdot \rho
$$

In quantum mechanics a so-called probability density and probability current is given by the expressions ${ }^{5}$ :

$$
\begin{aligned}
\rho & =|\psi|^{2} \\
j & =\frac{\hbar}{2 m i}\left[\psi^{*}(\nabla \psi)-\left(\nabla \psi^{*}\right) \psi\right]
\end{aligned}
$$

So it is straight forward to interpret Eqn. 1 tentatively as an equation of motion for "quantum particles":

$$
\begin{aligned}
\frac{d x}{d t} & =\frac{j}{\rho} \\
& =\frac{\nabla S}{m}
\end{aligned}
$$

The last line follows, if one writes $\psi=R e^{\frac{i}{\hbar} S}$ and substitutes this into the definition of $j$-i.e. the phase $S$ is guiding the particle motion of a system

\footnotetext{
${ }^{4}$ Bohm's approach [8] stressed the importance of what he called the quantum potential - our presentation sticks more to the road favored by Bell $[3,4,5]$

${ }^{5}$ Of course our motivation of Bohmian mechanics assumes some familiarity with ordinary quantum mechanics and does not serve as a first contact to the subject of quantum phenomena.
} 
that is described by the wavefunction $\psi$. The definition of $j$ is chosen to ensure the following continuity equation ${ }^{6}$ :

$$
\frac{\partial \rho}{\partial t}+\nabla j=0
$$

Hence, once the particle density of a system described by the wavefunction $\psi$ is $\rho=|\psi|^{2}$ distributed in position space, it will stay so. In other words: Given a $|\psi(x, 0)|^{2}$ distribution as the initial condition for the particle trajectories, they will produce the very same predictions as ordinary quantum mechanics with the probability rule postulated by Born [10]. One can programme a computer to integrate Eqn. 3 for arbitrary solutions of the Schrödinger equation $^{7}$. Let us look at the famous double slit experiment, which - as we were all told - allows no explanation in terms of particles moving on trajectories. The result for some trajectories is displayed in Fig. 1 and shows exactly the quantum mechanical interference pattern since (i) the wavefunction interferes at the double slit and is "guiding" the particles as described by Eqn. 3 and (ii) the initial conditions of the particles in front of the slits are distributed according to $\rho=|\psi|^{2}$.

But what about our earlier claim that Bohmian mechanics allows for the derivation of Born's rule (i.e. the postulate that $|\psi|^{2}$ is the probability density for measuring particles in a given volume) rather than postulating it? Antony Valentini has worked out a dynamical explanation $[15,16]$, how arbitrarily distributed configurations emerge into a $|\psi|^{2}$ "equilibrium". This mechanism has some resemblance to the corresponding problem in thermodynamics ${ }^{8}$. In any event it is not possible to prepare a system more precisely than according to the $\rho=|\psi|^{2}$ distribution which degrades the determinism of Bohmian mechanics to an in-principle one ${ }^{9}$. But that is very much like the situation in statistical physics, where one still entertains the notion of in-principle deterministic motion although nobody tries seriously to follow the path of a single particle.

It should be commented on two peculiarities of the Bohmian trajectories: (i) as can be seen in Fig. 1 they do show completely unclassical behavior

\footnotetext{
${ }^{6}$ In the standard treatment referred to as conservation of probability

${ }^{7}$ Our guiding equation 3 is formulated for the 1-particle case. The generalization to $\mathrm{N}$-particles and particles with spin etc. exists and is straight forward [11]

${ }^{8}$ There exist in fact different strategies to clarify the status of the so-called "quantum equilibrium", see e.g. [12].

${ }^{9}$ In this sense the deterministic trajectories are like the aether in the Lorentz interpretation undetectable
} 


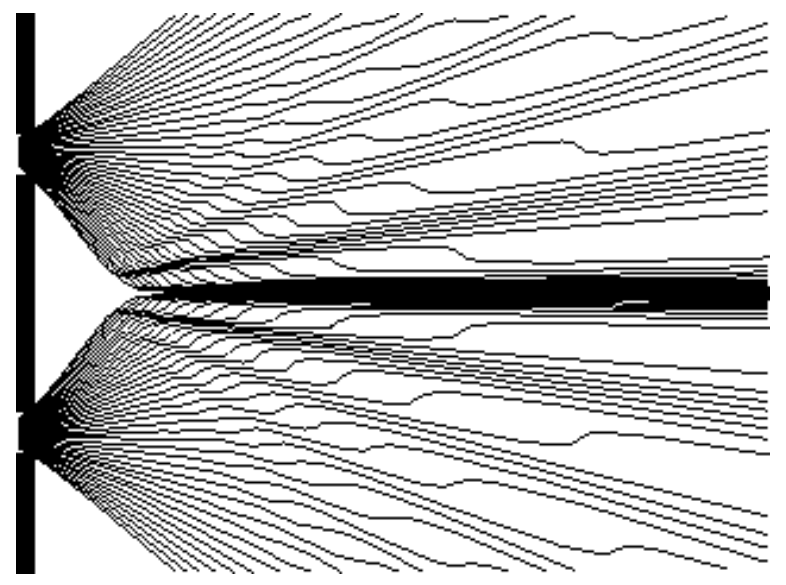

Figure 1: A numerical simulation of a sample of Bohmian trajectories for the double slit experiment [14]. Continuous and deterministic trajectories lead to the well known pattern, since they are "guided" by the wavefunction, which interferes at the double slit.

like kinks in the "field-free" region between double-slit and screen. But that is just to say, that they follow a Bohmian and not a Newtonian mechanic. Since the guiding equation 3 is first order, classical concepts like momentum, work or energy lose their relevance on the level of individual trajectories. (ii) Bohmian mechanics is said to be manifestly non-local, i.e. the motion of any particle is connected to the position of all other particles, since the wavefunction is defined on the configuration space - as opposed to position space. But it is exactly this non-locality, which allows Bohmian mechanics to violate the Bell inequalities [6] as demanded by experiment. More over the impossibility to prepare systems more precisely than $|\psi|^{2}$ distributed makes sure that signal-locality is obeyed [15]. For the same reason Bohmian mechanics does also not allow for an experimental violation of Heisenberg's uncertainty principle [15].

For the adherent of Bohmian mechanics it has the virtues to offer a clear ontology - as opposed to the concept of complementarity - and to provide an elegant solution to the notorious measurement problem of quantum mechanics (see e.g. [17]). In a nutshell the measurement problem consists of how to interpret a superposition of macroscopic distinct objects (like pointers, cats or the like). Ordinary quantum theory has to struggle hard to come up with a reasonable solution for how to bring together the unitary time evo- 
lution of the Schrödinger equation with the seemingly spontaneous collapse during measurement - in fact there is no universally accepted way how to resolve this problem. The poor man's solution being to adopt an ensemble interpretation of quantum mechanics [9], which denies the applicability of quantum mechanics to individual events. Within Bohmian mechanics the problem dissolves, since the system is now described by the pair of wavefunction and configuration in position space. The continuous trajectories select the branch of the superposition which will be measured - an elegant way to get rid of collapse when describing single measurements. But again: in the absence of any detailed control over the initial conditions beyond the $|\psi|^{2}$ quantum-equilibrium-distribution, this solution of the measurement problem does not enlarge the predictive power of the theory.

If one therefore adopts a merely positivistic attitude, Bohmian mechanics and ordinary quantum theory are completely equivalent since no experiment can discriminate between them. But people who feel a stronger commitment to ontology and scientific realism have trouble to come up with a criteria for which one to prefer ${ }^{10}$.

Bell finishes his paper with a comparison between special relativity and the Lorentzian aether theory which can be easily adapted to our case:

The approach of Bohr, Heisenberg, Pauli and many others differs from that of Bohm and de Broglie in two major ways. There is a difference of philosophy, and a difference in style. The difference in philosophy is this. Since it is experimentally impossible to predict any single outcome of a quantum process (say, which part of the screen behind the double slit will be hit next), the standard view declares probability as a "irreducible fact of the laws of nature" (W. Pauli). Bohm, on the other hand, preferred the view that there is indeed a deterministic substructure, even though the laws of physics conspire to prevent us from identifying it experimentally. The facts of physics do not oblige us to accept one philosophy rather than the other. And we need not accept Bohm's philosophy to accept a Bohmian pedagogy. Its special merit is to drive home the lesson that it is still possible to entertain consistently the notion of quantum particles moving on deterministic trajectories if we are willing to accept a non-local dynamic and put away the classical prejudice that these particles move on a straight path in "field-free" space - free, that

\footnotetext{
${ }^{10}$ The serious question of a relativistic generalization of Bohmian mechanics is for sure among the important criteria for judging the impact Bohmian mechanics. This problem is the subject of current research, see e.g. [13]. Similarly the generalization of the aether theory to general relativity is problematic.
} 
is, from fields other than the de Broglie-Bohm.

The difference in style is that instead of inferring quantum phenomena from known and conjectured laws of physics, the standard presentation has a stronger emphasis on postulates and axioms. This permits a very elegant formulation ${ }^{11}$ as often happens when a few big assumptions can be made to cover several less big ones. There is no intention here to make any reservation whatever about the power and precision of the standard approach. But in my opinion there is also something to be said for taking students along the road made by de Broglie and Bohm ${ }^{12}$. The longer road sometimes gives more familiarity with the country.

In connection with this paper I warmly acknowledge the assistance of Roderich Tumulka for teaching Bohmian mechanics to me and Jeremy Butterfield for valuable comments when preparing this note.

\section{References}

[1] J. S. Bell, How to Teach Special Relativity, Progress in Scientific Culture, Vol. 1, No. 2 (1976), reprint in [2].

[2] J. S. Bell, The Foundation of Quantum Mechanics, M. Bell, K. Gottfried and M. Veltman (eds.) World Scientific, (2001).

[3] J. S. Bell, de Broglie-Bohm delayed-choice, double-slit experiment, Int. J. Quantum Chem.: Quantum Chem. Symp. 14 (1980), reprint in [2].

[4] J. S. Bell, Quantum Mechanics for Cosmologists, in Quantum Gravity, C. Isham, R. Penrose and D. Sciama (eds.), Oxford University Press (1981), reprint in [2].

[5] J. S. Bell, On the impossible pilot wave, Foundation of Physics, Vol. 12, (1982), reprint in [2].

[6] J. S. Bell, On the Einstein Podolsky Rosen Paradox, Physics, Vol. 1, No. 3 (1964), reprint in [2].

\footnotetext{
${ }^{11}$ leaving aside for a moment the debatable questions whether e.g. the measurement problem can be accounted for convincingly within standard quantum mechanics.

${ }^{12}$ Among the few modern textbooks taking essentially this road is that of James $\mathrm{T}$. Cushing [11].
} 
[7] H. R. Brown and O. Pooley, The origin of the spacetime metric: Bell's 'Lorentzian pedagogy' and its significance in general relativity, in Physics meets Philosophy at the Planck Scale, C. Callender and N. Huggett (eds.), in Cambridge University Press (1999), e-Print Archive: grqc/9908048.

[8] D. Bohm, A suggested interpretation of the quantum theory in terms of "hidden variables", Phys. Rev., 85, 166(I) und 180(II) (1952), reprint in $[17]$.

[9] L. E. Ballentine, The Statistical Interpretation of Quantum Mechanics, Rev. Mod. Phys. 42,4 (1970) 358.

[10] M. Born, Zur Quantenmechanik der Stossvorgänge., Z. Phys. 37, (1926) 863, English version: On the quantum mechanics of collisions, in [17], pp. $52-55$.

[11] James T. Cushing, Quantum Mechanics - Historical Contingency and the Copenhagen Hegemony, University of Chicago Press (1994).

[12] D. Dürr, S. Goldstein and N. Zanghi, Quantum equilibrium and the Origin of Absolute Uncertainty, Journal of Statistical Physics, 67 (1992) 843.

[13] K. Berndl et al., Nonlocality, Lorentz Invariance, and Bohmian Quantum Theory, Phys. Rev. A 53, (1996) 2062-2073.

[14] C. Philippidis, C. Dewdney and B. J. Hiley, Quantum Interference and the Quantum Potential, Il Nuovo Cimento 52B (1979) 15.

[15] A. Valentini, Signal-locality, uncertainty, and the subquantum Htheorem. I, Physics Letters A 156, No.1-2, (1991) 5.

A. Valentini, Signal-locality, uncertainty, and the subquantum $H$ theorem. II, Physics Letters A 158, No.1-2, (1991) 1.

[16] A. Valentini, Universal Signature of Non-Quantum Systems, quantph/0309107

[17] J. A. Wheeler und W. H. Zurek (eds.), Quantum Theory of Measurement, Princeton University Press, Princeton NJ, (1983). 